\title{
SHORT PAPER
}

EDDY MIXING COEFFICIENT ON SATURN

\author{
S. K. Atreya \\ Department of Atmospheric and Oceanic Science \\ Space Physics Research Laboratory \\ The University of Michigan \\ Ann Arbor, Michigan 48109, USA
}

(Received 24 May 1982)

\section{Abstract}

Data on the composition and thermal structure, and the Lyman-alpha dayglow of Saturn when analyzed in conjunction with photochemical models of the hydrocarbons and the atomic hydrogen production yield the homopause value of the eddy diffusion coefficient to be approximately $10^{8} \mathrm{~cm}^{2} \mathrm{~s}^{-1}$. The equatorial value of the eddy diffusion coefficient at the homopause of Saturn is thus found to be approximately 100 times greater than on Jupiter. The mesosphere (and presumably, troposphere) of Saturn appears to be considerably more turbulent than the upper atmosphere of Jupiter.

\section{INTRODUCTION}

In an earlier paper, we discussed the aeronomical implications, including atmospheric mixing and energy budget, of the upper atmospheric composition and thermal structure of Jupiter (Atreya, Donahue, and Festou, 1981). With similar data for Saturn now analyzed (Festou and Atreya, 1982), it is possible to determine the eddy diffusion coefficient on Saturn. As discussed by Festou and Atreya (1982), the upper atmospheric composition and temperature structure of Saturn were obtained by monitoring the tangent rays from a star Dzuba ( $\delta$-Scorpii), as they passed through the atmosphere of Saturn and viewed by an Ultraviolet Spectrometer aboard Voyager 2. In this paper, we discuss the determination of the eddy mixing coefficient by comparing the upper atmospheric methane abundance with theoretical models involving the ultraviolet photolysis of methane in the equatorial region. The technique is similar to the one employed successfully for determining the eddy mixing coefficient on Jupiter (Atreya, et al., 1981). We also discuss an alternate approach whereby the column abundance required to produce the observed Lyman-alpha airglow is compared with a theoretical model which relates such abundance to the eddy mixing coefficient at the homopause. The results of these two approaches are found to be quite similar. In both approaches, equatorial data are employed for comparison with the theoretical models, thus preventing complications due to charged particle destruction of methane and molecular hydrogen.

\section{MODEL}

Photolysis of methane occurs shortward of $1600 \AA$-- principally at the wavelength of the solar Lyman-alpha, $1216 \AA$. The distribution of methane $\left(\mathrm{CH}_{4}\right)$, ethane $\left(\mathrm{C}_{2} \mathrm{H}_{6}\right)$, acetylene $\left(\mathrm{C}_{2} \mathrm{H}_{2}\right)$, and ethylene $\left(\mathrm{C}_{2} \mathrm{H}_{4}\right)$ and other minor products can then be determined by considering a chemical scheme involving photochemical production and loss of these species, as has been discussed by Atreya, et al. (1981) for Jupiter. To initiate the computer program, boundary values for the mixing ratios of the relevant hydrocarbons and other species in the troposphere/ stratosphere of Saturn were taken from the Voyager infrared measurements (Hanel, et al., 1981 and 1982; Maguire, 1981). The relevant volumetric mixing ratios are: $\mathrm{He}, 0.06 ; \overline{\mathrm{CH}}_{4}, 8 \mathrm{x} 10^{-4}$; $\mathrm{C}_{2} \mathrm{H}_{6}, 5 \times 10^{-6} ; \mathrm{C}_{2} \mathrm{H}_{2}, 2 \times 10^{-8}$. The atmospheric temperature structure was taken from Festou and Atreya (1982). The temperature in the region where most of the photolysis and transport of methane occurs is $140 \mathrm{~K}$. The unly uther parameter of significance in the model calculations 


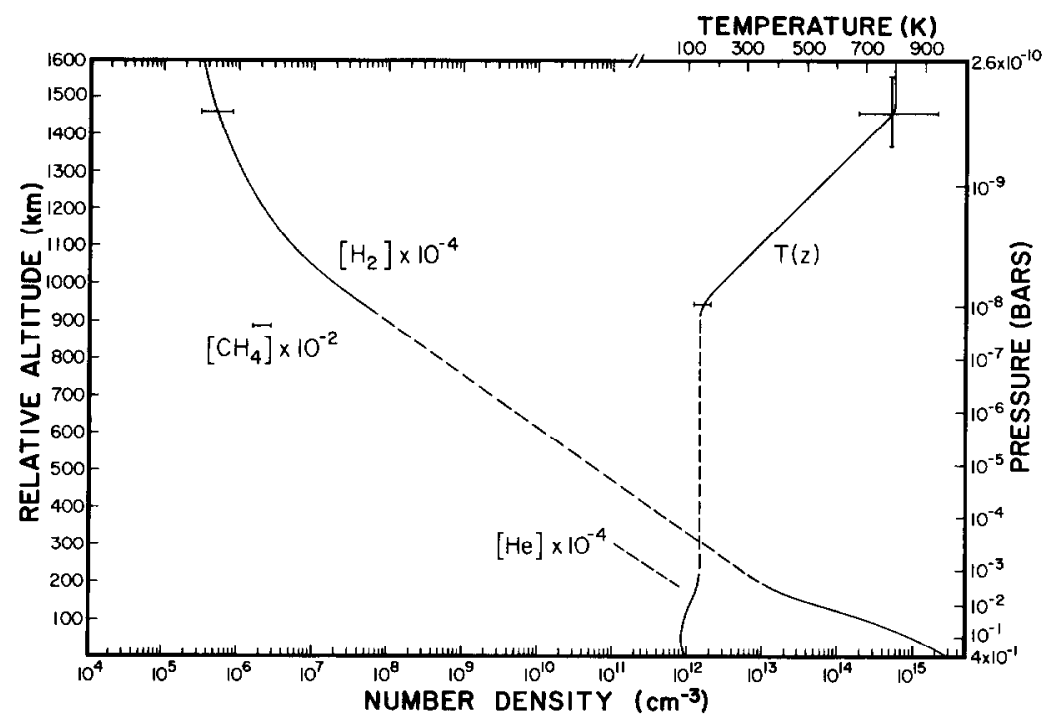

Fig. 1. Temperature and density profiles in Saturn's atmosphere. Information above approximately $890 \mathrm{~km}$ is obtained from the $\delta-5 c o$ exit occultation data, and below $200 \mathrm{~km} f r o m$ Voyager infra-red and radio science investigations for the equatorial region. The interpolation between $200 \mathrm{~km}$ and $890 \mathrm{~km}$ assumes a constant temperature of $140 \mathrm{~K}$. The $6 \%$ volume fraction of helium determined by the IR technique is illustrated by a broken line in the homosphere. The zero of the altitude scale is at the ammonia cloud tops located at approximately 0.4 bar, 97 K 1 evel.

is the eddy diffusion coefficient, $K$. The value of $K$ was fixed at the homopause to be $10^{5}$, $10^{6}, 10^{7}$, and $10^{8} \mathrm{~cm}^{2} \mathrm{~s}^{-1}$ (homopause in this exercise is defined as the level at which the molecular diffusion coefficient of a given species, here $\mathrm{CH}_{4}$, equals the eddy diffusion coefficient. Although several variations of $\mathrm{K}$ with altitude were attempted, the most suitable was found to be the one in which Kvaried inversely as the square root of the atmospheric number density -- as was also the situation with Jupiter (Atreya, et a1., 1981). Although the photochemical program yields height distributions of all the hydrocarbons and atomic hydrogen, we show in Figure 2 the distribution of only $\mathrm{CH}_{4}$ since it is the only hydrocarbon species whose abundance has becn detcrmined with confidence in the analysis of the $\delta-S c o r p i i$ data (Figure 1). Furthermore, as was the case with Jupiter, only $\mathrm{CH}_{4}$ enters the analysis that yields the value of the eddy mixing coefficient.

The $\delta$-Sco occultation experiment yields a methane density of $1.9 \times 10^{8} \mathrm{~cm}^{-3}$ at an altitude where the $\mathrm{H}_{2}$-density is $1.2 \times 10^{12} \mathrm{~cm}^{-3}$ (Figure 1 , Festou and Atreya). In the photochemical calculations shown in Figure 2, a methane density of $1.9 \times 10^{8} \mathrm{~cm}^{-3}$ is reached at altitudes where the $\mathrm{H}_{2}$-density is: $6 \times 10^{14} \mathrm{~cm}^{-3}$ for $\mathrm{K}=10^{5} \mathrm{~cm}^{2} \mathrm{~s}^{-1} ; 4 \mathrm{x} 10^{13} \mathrm{~cm}-3$ for $\mathrm{K}=10^{6} \mathrm{~cm}^{2} \mathrm{~s}^{-1} ; 9 \times 10^{12} \mathrm{~cm}^{-3}$ for $\mathrm{K}=10^{7} \mathrm{~cm}^{2} \mathrm{~s}^{-1}$; and $1.6 \times 10^{12} \mathrm{~cm}^{-3}$ for $\mathrm{K}=10^{8} \mathrm{~cm}^{2}$ $\mathrm{s}^{-1}$. Thus, a comparison of the model calculations with the data in Figure 1 indicates that $K=10^{8} \mathrm{~cm}^{2} \mathrm{~s}^{-1}$ case gives results closest to the obscrvations. Actually, an eddy diffusion coefficient slightly greater than $10^{8} \mathrm{~cm}^{2} \mathrm{~s}^{-1}$ would be implied, if an exact match between the model and data were sought. Instead of carrying out such fine tuning in the model, we have resorted to the following more quantitative approach which has already been tested successfully for Jupiter (Atreya, et al., 1981). 


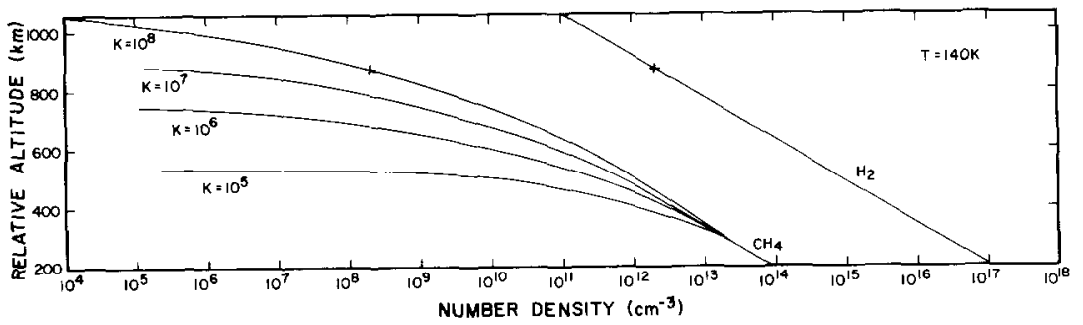

Fig. 2. Distribution of methane and $\mathrm{H}_{2}$-density as a function of altitude for the hompause values of eddy diffusion coefficient of $10^{5}, 10^{6}, 10^{7}$, and $10^{8} \mathrm{~cm}^{2} \mathrm{~s}^{-1}$. $\mathrm{K}$ is assumed to vary inversely as the square root of the atmospheric number density. In the $\delta-S c o$ data (Fig. 1), a $\mathrm{CH}_{4}$-density of $1.9 \times 10^{8} \mathrm{~cm}^{-3}$ occurs at an altitude where the $\mathrm{H}_{2}$-density is $1.2 \mathrm{x} 10^{12}$ $\mathrm{cm}^{-3}$. In this photolysis calculation, $\mathrm{CH}_{4}$-density of $1.9 \times 10^{8} \mathrm{~cm}^{-3}$ occurs at an altitude where the $\mathrm{H}_{2}$-density is $1.6 \times 10^{12} \mathrm{~cm}^{-3}$ (shown by crosses) for $\mathrm{K}=10^{8} \mathrm{~cm}^{2} \mathrm{~s}^{-1}$. Thus $\mathrm{K}=$ $10^{8} \mathrm{~cm}^{2} \mathrm{~s}^{-1}$ case most closely reproduces the observed $\mathrm{CH}_{4}-\mathrm{H}_{2}$ density combination. The altitude scale is same as in Figure 1.

$\uparrow$ By determining the altitude at which the vertical optical depth of methane at Lymanalpha, $\mathrm{TH}_{4}$, reaches unity for the various eddy diffusion coefficients, and then comparing this altitude with the altitude of $\tau_{\mathrm{CH}}^{\uparrow}=1$ in the data, we have been able to determine the homopause eddy diffusion coefficient ${ }^{4}$ precisely. We show in Figure 3 the density of $\mathrm{H}_{2}$ at the $\mathrm{ICH}_{4}=1$ level as a function of the eddy diffusion coefficient at the homopause; these results are a by-product of the abovementioned methane photolysis program.

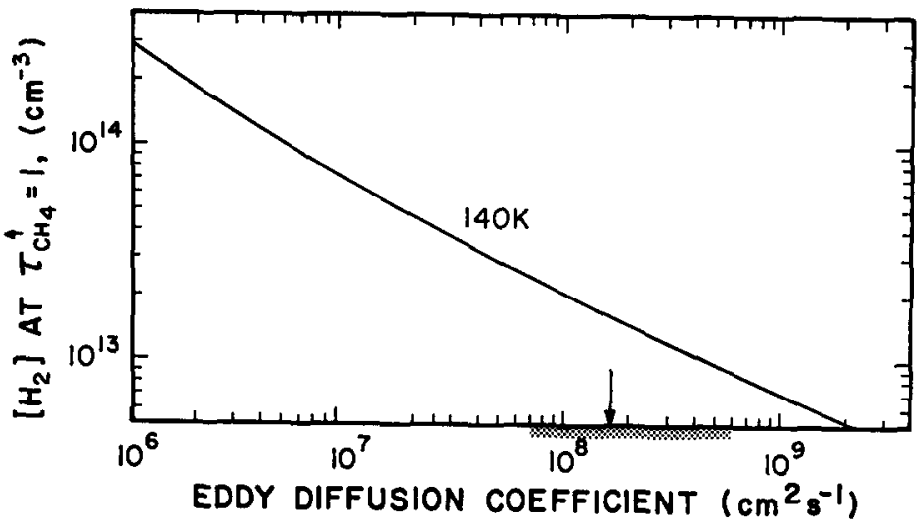

Fig. 3. $\mathrm{H}_{2}$-density at the altitude of unit vertical optical depth in methane at Lyman-alpha $\underline{v s}$. eddy diffusion coefficient at the homopause, $\mathrm{K}_{\mathrm{h}}$. The arrow represents the value of $\mathrm{K}_{\mathrm{h}}$ corresponding to the 'central' value of the $\mathrm{H}_{2}$-density at the $\mathrm{T}_{\mathrm{CH}_{4}}=1$ level determined from the $\delta$-Sco occultation data. The shaded area on the X-axis currespunds to statistical uncertainty in the determination of the $\tau_{\mathrm{CH}}^{+}=1$ altitude level in the data. 
The $\$$-Sco data (Figure 1 ) yield $\tau^{\uparrow}=1$ at an altitude where the $\mathrm{H}_{2}$ äensity is $1.6 \times 10^{13} \mathrm{~cm}^{-3}$ (Festou and $\mathrm{Atreya}$ ). For $\mathrm{CH}_{4}$ this $\mathrm{H}_{2}$-density, an eddy diffusion coefficient of $1.7 \times 10^{8} \mathrm{~cm}^{2} \mathrm{~s}^{-1}$ at the homopause is implied, as is indicated by an arrow in Figure 3. This is the 'central' value of the eddy diffusion coefficient. The statistical uncertainty in the determination of the $\tau \mathrm{CH}_{4}=1$ level in the data is, however such that $\mathrm{T}^{+}=1$ occurs at altitudes bounded by the following, $\mathrm{H}_{2}$-densities: $9 \times 10^{12} \mathrm{~cm}^{-3}<\mathrm{H}_{2} \mathrm{CH}_{4}{ }^{2} .5 \times 10^{13}$ $\mathrm{cm}^{-3}$, implying $6 \times 10^{8} \mathrm{~cm}^{2} \mathrm{~s}^{-1}>\mathrm{K}>7 \times 10^{7} \mathrm{~cm}^{2} \mathrm{~s}^{-1}$. This statistical range of the homopause eddy mixing coefficient on saturn 1 s shown by the shaded area in Figure 3.

Finally, we discuss the approach in which the planetary Lyman-alpha emission is exploited to determine the eddy mixing. Unlike the earlier work of Wallace and Hunten (1973), our calculations assume the appropriate high exospheric temperature on Saturn, and include photolysis and transport of methane along with coupling with the ionosphere. The calculations assume no contribution to the hydrogen abundance from the auroral charged particledissociation of $\mathrm{H}_{2}$. It is certainly an appropriate assumption for the equatorial Lyman-alpha on Saturn as has been shown by calculations of J. H. Walte, Jr., and discussed elsewhere (Atreya, et al., 1982). It is found that with either a $1 \mathrm{keV}$ or $10 \mathrm{keV}$ auroral beam with

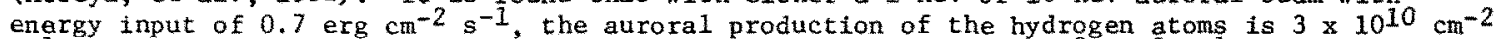
$\mathrm{s}^{-1}$; the EUV and phtotoelectron hydrogen production rate is $3.9 \times 10^{9} \mathrm{~cm}^{-2} \mathrm{~s}^{-1}$. The aurora1 production rate of H-atoms, although ten times greater than the EUV and photoelectron H-prom duction rates combinea, is confined to a narrow latitude range of approximately $3^{\circ}$, from $78^{\circ}$ - 81.5\% (Sande1, et al., 1982), thus making a negligible contribution to the average nonauroral abundance of hydrogen atoms. Therefore, unlike in the case of Voyager/Jupiter, the equatorial Lyman-alpha emission on Saturn is excited by the solar resonance scattering of the hydrogen atons produced by EUV and photoelectrons alone (indeed, the abovementioned calculations of $\mathrm{J}$. H. Waite, Jr., yield $\mathrm{H}$-abundance in the equator to be $3 \times 10^{16} \mathrm{~cm}^{-2}$, a value exceedingly close to what is needed to produce the observed Lyman-alpha, see below).

We show in Figure 4 the results of calculations which relate the hydrogen column abundance above the $\tau=1$ level in methane to the assumed values of the homopause eddy diffusion coefficient. The non-auroral central-disc intensity of Lyman-alpha on Saturn is

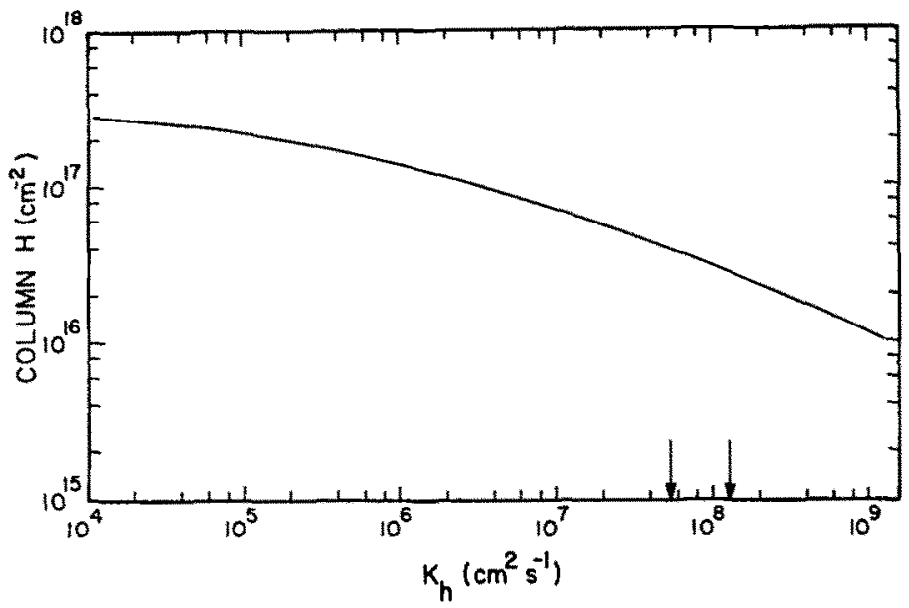

Fig. 4. Colum abundance of atomic hydrogen above the unit optical depth 1 evel in methane as a function of the homopause value of the eddy mixing coefficient. These calculations assume the appropriate atmospheric temperature structure and the solar fluxes. The arrows represent the situations with and without the contributions of the interplanetary/interstellar Lymanalpha backscatterigg to the observed planetary Lyman-alpha airglow. The corresponding values of $\mathrm{K}_{\mathrm{h}}$ are $1.4 \times 10^{8} \mathrm{~cm}^{2} \mathrm{~s}^{-1}$ and $8 \times 10^{7} \mathrm{~cm}^{2} \mathrm{~s}^{-1}$ respectively (adapted fron Waite, 1981 ). 
$3 \mathrm{kR}$ (Sande1, et a1., 1982) requiring an atomic hydrogen abundance of $4 \times 10^{16} \mathrm{~cm}^{-2}$ above the methane absorbing layer. From Figure 4, we determine an eddy mixing coefficient of $8 \times 10^{7} \mathrm{~cm}^{2} \mathrm{~s}^{-1}$ for this column abundance. Sandel, et al. (1982) reached the same conclusion from a preliminary analysis of the Satumfan Lyman-alpha. The observed 3 kR Lyman-alpha intensity is, however, expected to include a small contribution of approximately $0.5 \mathrm{kR} f$ rom the backscattering of the interplanetary/interstellar hydrogen Lyman-alpha. Thus, only about $2.5 \mathrm{kR}$ could be attributed to the planetary Lyman-alpha, then only $2.7 \times 10^{16} \mathrm{~cm}^{-2} \mathrm{hydrogen}$ abundance would be required. This would then result in an eddy diffusion coefficient of $1.4 \times 10^{8} \mathrm{~cm}^{2} \mathrm{~s}^{-1}$ at the homopause (Figure 4).

\section{DISCUSSION}

All three methods discussed above yield the central value of the homopause eddy diffusion coefficient on Saturn to be on the order of $10^{8} \mathrm{~cm}^{2} \mathrm{~s}^{-1}$. The extremes are bracketed by the analysis based on the $\tau \mathrm{CH}_{4}=1$ level determination, i.e., $7 \times 10^{7} \mathrm{~cm}^{2} \mathrm{~s}^{-1}<\mathrm{K}_{\mathrm{h}}<$ $6 \times 10^{8} \mathrm{~cm}^{2} \mathrm{~s}^{-1}$. A comparison with the values for the equatorial region of Jupiter $1 \mathrm{~s}$ shown in Table 1 .

\section{TABLE 1}

EDDY DIFFUSION COEFFICIENT

\begin{tabular}{|c|c|c|c|c|}
\hline & $\left(\mathrm{cm}^{2} \mathrm{~s}^{\mathrm{K}_{\mathrm{h}}}\right)$ & $\begin{array}{c}{\left[\mathrm{H}_{2}\right] \text { at the }} \\
\text { homopause } \\
\left(\mathrm{cm}^{3}\right)\end{array}$ & $\begin{array}{l}\text { Altitude of } \\
\text { homopause } \\
(\mathrm{km})\end{array}$ & $\begin{array}{l}\text { Atmospheric } \\
\text { pressure at } \\
\text { homopause } \\
\text { (bars) }\end{array}$ \\
\hline Saturn & $10^{8}$ & $1.2 \times 10^{11}$ & 1030 & $4 \times 10^{-9}$ \\
\hline Jupiter & $1.4 \times 10^{6}$ & $1.4 \times 10^{13}$ & 400 & $10^{-6}$ \\
\hline
\end{tabular}

It is evident from Table 1 that the upper mesosphere of Saturn is considerably more turbulent than the corresponding region on Jupiter. Since on both Jupiter and Saturn a similar variation of the eddy mixing with depth is envisioned, it is therefore implied that the tropospheric value of the eddy diffusion coefficient on Saturn is at least ten times greater than on Jupiter. The high value of the eddy diffusion coefficient in the upper atmosphere of Saturn is considtent with the observation of relatively cool mesospheric and lower thermospheric temperature compared to Jupiter at corresponding atmospheric pressures. Although it is not immediately obvious why Saturn should exhibit a greater eddy mixing than Jupiter, some ideas are put forth here. It could perhaps be related to the strong equatorial jets on Saturn. Smith, et al. (1981 and 1982) have reported equatorial prograde wind velocities which are nearly four times greater than the maximum observed on Jupiter. They are also greater in latitudinal extent than those on Jupiter. Such strong equatorial jets would tend to result in more violent eddy mixing on Saturn than on Jupiter, the effect is likely to propagate in the upper atmosphere. Another possibility is that in comparison to Jupiter, Saturn has a somewhat greater ratio of the emitted heat flux to the energy absorbed from the Sun, as concluded by Hanel (1982) and Ingersol1 (1980). The condensation and subsequent rainout of helfum to which some of the excess internal heat on Saturn has been attributed is likely to be a violent process and it could cause upward propagating small-scale turbulence. Also, one cannot discard the possibility of a different stratospheric/mesospheric temperature structure on Saturn than on Jupiter which is expected to be reflected in the nature of the lower atmospheric vertical mixing.

This research was sponsored by the Planetary Atmospheres Program of the NASA Office of Space Science and Applications.

I thank D.M. Hunten and P.B. Hays for valuable discussion. 


\section{References}

Atreya, S. K., Donahue, T. M. and Festou, M. C. (1981) Astrophys. J., 247, L43.

Atreya, S. K., et al. (1982) Review Chapter in Saturn (ed. T. Gehrels), University of Arizona Press, in preparation.

Festou, M. C., et a1. (1981) J. Geophys. Res., 86, 5715.

Festou, M. C. and Atreya, S. K. (1982) submitted to Geophys: Res. Lett.

Hanel, R., et al. (1981) Science, 212, 192.

Hane1, R., $\overline{\mathrm{et}} \overline{\mathrm{al}}$. (1982) Science, $\overline{215}, 544$.

Hane1, R. (1982) personal communication.

Ingersoll, A., et a1. (1980) Science, 207, 439.

Maguire, W. C. (1981) personal communication.

Sandel, B. (1982) Science, 213, 348.

Smith, B. A., et al. (1981) Science, 212, 163.

Smith, B. A., et al. (1982) Science, $\overline{\text { ZTS }}, 504$.

Waite, J. H., Jr. (1981) Ph.D. Thesis, University of Michigan.

Wallace, L. and Hunten, D. M. (1973) Astrophys. J., 183, 1013. 